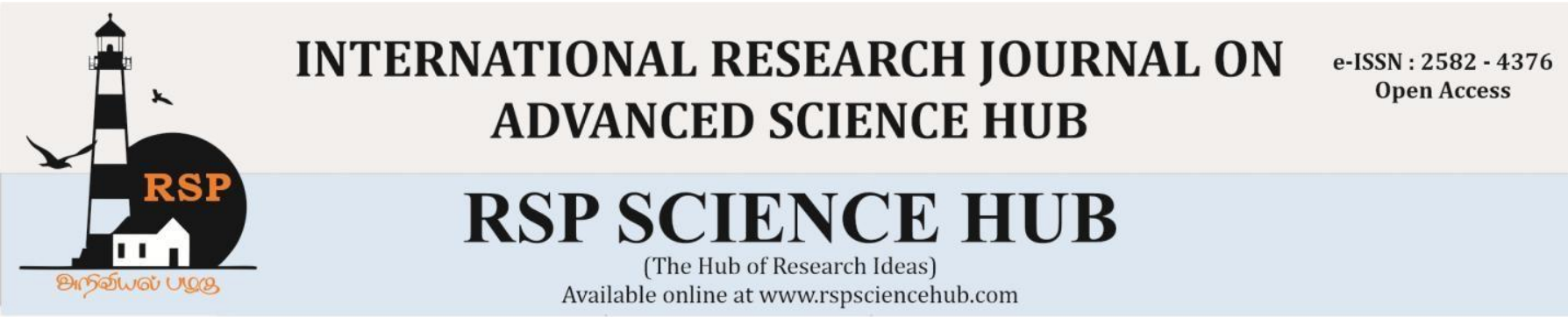

\title{
Review of Deployment of Machine Learning in Blockchain Methodology
}

Ms. Sona D Solanki ${ }^{1}$, Mrs. Asha D Solanki ${ }^{2}$

${ }^{l} P G$ Student, Department of Electronics and Communication Engineering, Babaria Institute of

Technology, Vadodara, India.

${ }^{2}$ Department of Arts, B. K. Arts and Science College, Palanpur, India.

solankisona28@gmail.com ${ }^{1}$

\begin{abstract}
The evolution of blockchain methodology has been a remarkable, highly transformative and trend-setting platform in current years. BT's accessible platform reinforces data protection and confidentiality. In addition, the consensus framework in it ensures system is protected and accurate. Nevertheless, it introduces additional security challenges such as invasion by the majority and double consumption. Data analysis on encrypted data centered on blockchain is crucial to manage the existing challenges. Insights on these results elevates the value of emerging of Machine Learning technique. It covers the fair quantity of data needed to make specific choices. Consistency of data and its distribution are very critical in ML to increase findings reliability. The fusion of these two techniques will produce extremely accurate outcomes. In this article, we describe a thorough analysis on ML implementation to make smart applications based on BT further robust to threats. There are numerous standard ML approaches such as Support Vector Machines (SVM), Clustering, Bagging, and Deep Learning (DL) algorithms such as Convolutional Neural Network (CNN) and Long-Term Memory (LSTM) that can be employed to evaluate the threats on a block chain network. Finally, we discuss how two different techniques can be implemented in a number of smart applications like Unmanned Aerial Vehicle (UAV), Smart Grid (SG), medical care and Smart cities.
\end{abstract}

Keywords: Blockchain, Machine Learning, Smart Implementation, Smart Grid, Data Analysis.

\section{Introduction}

Over the last couple of years, information has been an important resource of knowledge and brings new opportunities across smart solutions to actual-life issues such as wireless networking, biophysics, farming, and economics [1]. These implementations are information-driven and integrate implementable observations into a user experience that helps entities to more effectively achieve the required output. It incorporates information, modifies experience, enhances customer interactions and increases operating costs and effectiveness for the new product line.

There are numerous smart developments like Smart Grid (SG), Unmanned Aerial Vehicle
(UAV) and Smart Cities that render life simpler for a person. Such frameworks produce enormous quantities of information and storing this quickest evolving content in servers is a challenge as well as its interaction poses safety issues. BT (Blockchain technology) that has a decentralized server system is employed to manage such concerns. It was invented in 2008 by Satoshi Nakamoto and included a time-stamped set of manipulating evidence documents, which a network of decentralized machines operates. It is termed one of the Artificial Intelligence implementations. ML gives machines the skills to acquire through specific programming. Its specific concept is to create an effective method that can recognize source information, render a forecast 


\section{www.rspsciencehub.com}

and modify the outcomes with the aid of quantitative processing. It evaluates a large volume of details for making information-driven decisions. Protection concerns are treated layerwise within a connectivity infrastructure with BT dependent smart programs. On the network layer, certain vulnerability problems are addressed such as fraudulent packets and others at the application layer like malware.

Correspondingly, UAVs provide a substantially specific configuration of the system relative to the traditional configuration of the blockchain. It involves satellite connectivity and numerous ground sites. For

$\mathrm{UAV}, \mathrm{BT}$ is utilized to preserve co-ordinates and certain related information safely to preserve the vehicles' chart integrity. In corresponding articles, we discuss the notable analysis on the implementation of ML in the smart framework centered across the blockchain.

\section{Analysis of the Context}

Numerous investigators to date have release the numerous research analysis on the various elements of the implementation of ML in blockchain-focused smart implementations. As well as our awareness is concerned many analyses also concentrated on particular regions, sectors, or technologies in which both ML and blockchain are needed.

Then Ucci et al. used ML strategies to investigate vulnerability detection. Malware functionality was debated at length, and a detailed classification was suggested [3]. Salah et al. and Casino et al. examined programs centered on blockchain [4]. It comprises aims, benefits, and disadvantages of group evaluations about various variables such as design, implementations, and accessible questions, categorization, and benefits, disadvantages of current strategies. Figure 3 describes a basic categorization of ML for BT.

\section{Machine Learning}

ML is the analysis area that emphasizes on developing solutions that benefit from observation. It is the capacity to instruct a machine through directly scripting it. It incorporates its study across a range of fields involving philosophy, data analysis, prediction and probability, regulation theory, psychology and neuroscience, conceptual technology, and artificial intelligence [5]. Its programs are included in several utilizations, and have gained from the following:

ML implementation unique implementation and relies on the performance the device needs. A few of its implementations function, including Supervised, Semi-Supervised and Unsupervised ML. Utilizing predictive methods, supervised ML predicts performance in statistical information and classifies the right label [6]. The most widely identified techniques there comprise the methodology to analyze and the trees for decision. Unsupervised learning can be implemented to explore the input factors framework, whereupon it is employed to render effective forecasts for unidentifiable information. It incorporates information that was expected directly into the monitored ML algorithm as learning information and uses the model to predict unknown information.

\section{Blockchain}

Blockchain are an infinite collection of documents for inspection that are encryption connected. It is analogous to a financial list. Prior documents in the operating system cannot be modified now and a reliable entity has to check new information. The main distinction among such concepts is that a distributed network framework that has a BT version verifies different chains. Any central body does not check the documents. It is constructed by joining apart legitimate frames; the present block includes a former blockchain and thus seen in Figure 1.

It is processed in the blockchain as texts and implemented to generate outcomes that are required from the original contract due to the information provided to them. It controls processes that are whether performed in whole or in portion centered on the existing data. A smart contract's central aim is to establish outstanding protection by reducing costs and the limitations correlated with standard contracting.

\section{Incorporation of Machine Learning in Blockchain Centered Systems}

ML's thinking features can be extended to programs centered on blockchain to render their smarter. Through utilizing decentralized ledger ML, protection can be enhanced. It can even be considered for creating efficient information exchange links to improve the period required to achieve a compromise. Furthermore, by leveraging control of BT's distributed structure it generates an incentive to develop appropriate 


\section{www.rspsciencehub.com}

designs. In the BT-centered smart system, we recommended a framework for their implementation, as demonstrated in Figure 2. There, the smart application gathers information through various information resources like sensors, mobile machines, and computers leveraging the Internet of Things (IOT).

This eliminates information failures like replication, incomplete information quality, mistakes and interference. It concentrates on the information and thus information-based problems can be removed in $\mathrm{ml}$ frameworks. ML-models may be based on individual chain segments instead of the whole information-set.

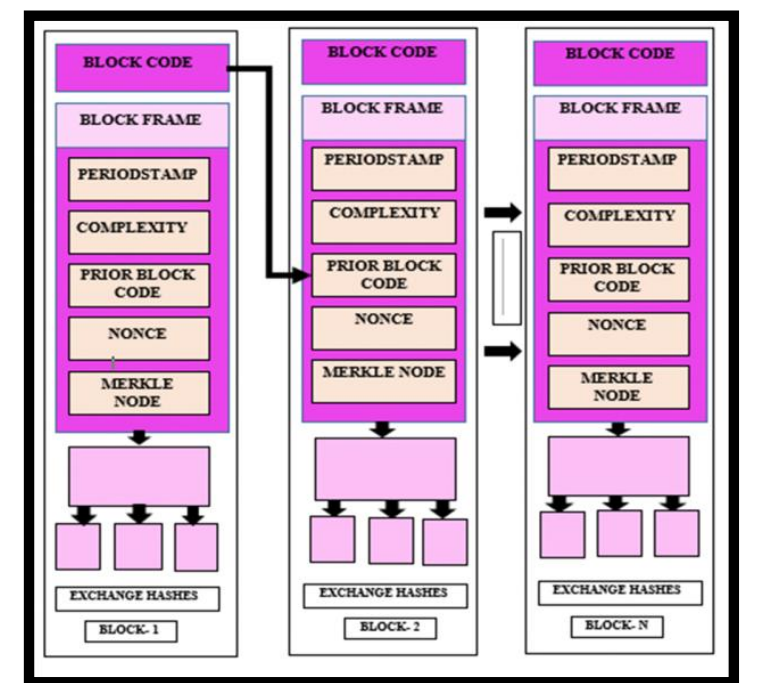

Fig 1. Framework of Blockchain

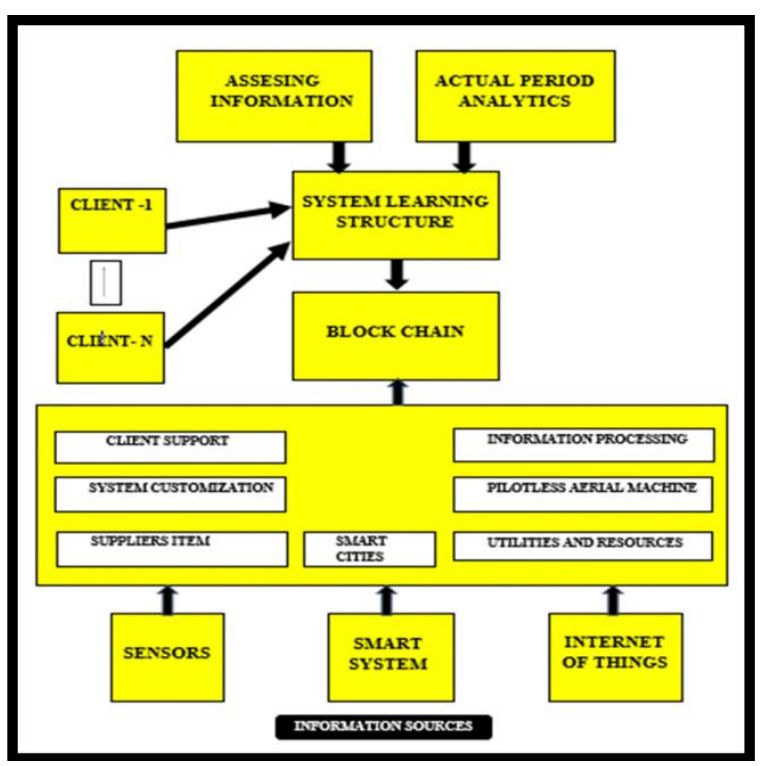

Fig 2. Structure for Machine Learning deployment in Blockchain centered Smart Implementation.

It might offer unique designs for various
Volume 02 Issue 09 September 2020

implementations like detecting manipulation and identifying information fraud. Little of the gain is seen below if ML is implemented:

- Identification of the client as a legal client to demand or execute some operation inside the blockchain framework.

- BT offers a broad quality of safety and faith.

- Manipulates evidence smart agreements can be tested on different machines (offering separate hardware architectures), ML algorithms will not deviate towards their capacity and will deliver results just as they should.

- Transactions performed on a blockchain system in an actual moment, with confidence.

- For illustration, blockchain resources Ethereum work with hundreds of distributed devices across the globe. This has reassured consumers that it is neither entirely unachievable nor unavailable.

\section{Strategic Research Review}

Traditional repositories and Google Scholar are utilized to check for relevant analysis study leveraging terminology like "ML for Blockchain centered Smart Implementation". Articles focus mainly on blockchain solutions for smart implementations in a particular process. After this, developers focused on ML response for blockchain-centered smart technologies at the next step of the search. Centered upon the findings of such investigation, the extraction of redundant reports and acquiring the initial collection greater than 353 papers was done. Finally, merely 60 articles were chosen to address the categorization as indicated in Figure 3. where the component is color-coded to configure the categorize standard. For instance, the source reflects the graded categorization level 0 and is represented in green. Similarly, in yellow color, the four main parameters are defined as level 1, etc.

\section{Categorization}

The articles were reviewed in a document that is primarily focused on the $\mathrm{ml}$, blockchain respectively. The research provided was split into two aspects: preventive measures and smart implementations. Figure 3 demonstrates the $\mathrm{ml}$ deployment categorization for smart implementations that are centered on the blockchain. 


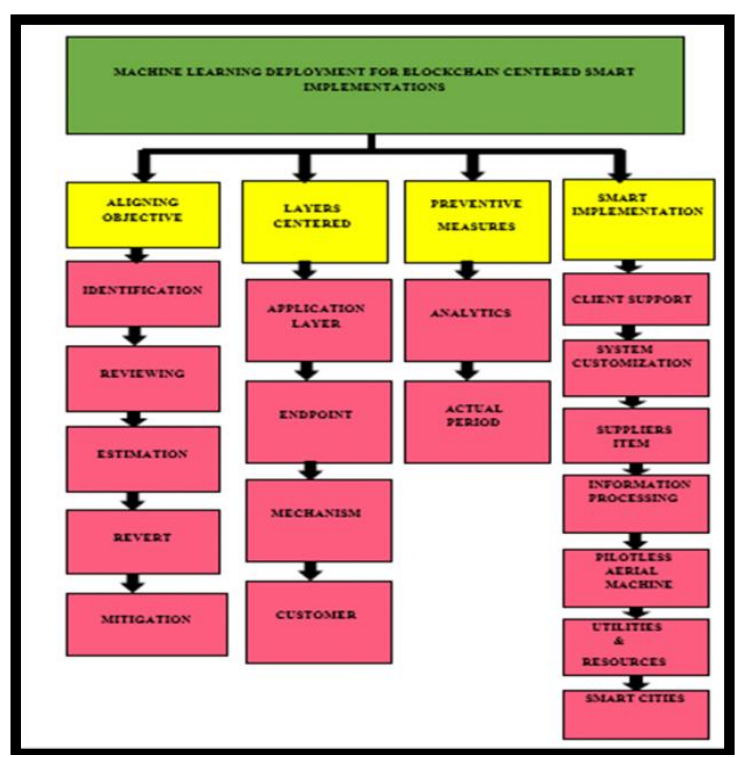

Fig 3. Categorization of ML deployment in Blockchain centered Smart Implementation.

\section{Preventive Measures}

Concerning a vulnerability or analysis of a device, the ML addressed can be described as an actualtime evaluation centered on statistical details

\subsection{Analytics}

Each business produces enormous quantities of information through various resources like digital networks, mobile handsets, IOT and many software machines in the current era. Such statistics are of great importance to organizations. A general method of extracting a consistent trend from such results is termed information processing. It is a mechanism of translating details through foresight to perspective. To encourage optimization, the cognitive learning technique works. Our culture must be powered by emerging technical enhancements in ML and BT in the coming generations. In addition, by employing the Artificial Neural Network (ANN) approach, a tracking technique has also been suggested for bitcoin processing [7]. The objective was to eliminate insignificant mining information and incorporate tracks into a structure. It was effective and reduced standardization duration in a distributed system. Deep Learning (DL) is a subclass of ML derived from authoritative systems that are inspired by the overarching framework and purpose of the human brain neural system.
There were input, outcome, and concealed numerous layer DL systems. It acknowledged a large structure and a preceding block background as an input in implementations centered on BT. On the functions like hash value, nonce, domain, and transaction information, using a stateconversion method. A classified methodology focused on ML is employed to classify the Bitcoin hacker-crime. The findings gave the Bitcoin community a preview of the scale of cyber threats.

\subsection{Actual Period}

ML offers actual period monitoring with certain information forms, such as digital-accessible, interactive and functional information. Although maintaining unrelentingly modified information safely, it employs in-memory programming. It enhances the precision of analysis and enhances ML systems' analytical conduct.

This structure incorporates the application of depth validation research and Ethereum blockchain. Ethereum networks were divided into two groups to preserve and exchange data: processing networks and non - resource entities. Three basic elements, behavior, conditions and incentives were used in the suggested Intense Reinforcing Learning method. Similar to a conditional approach, the recommended method demonstrates a $34.5 \%$ enhancement in regional justice.

\section{Smart Implementations}

As illustrated in Figure 4, the final aspect involves the implementation of ML strategies for BTcentered smart technologies like information processing, UAV, material processing, clinical and healthcare, smart cities, digital client support, and system customization. Both make headway in the big smart assessment:

\subsection{Client Support}

Client support requires being highly effective and streamlined to meet rising client demands with the client's resurgence. The popular approach is to optimize the plan to enhance the strengths of a firm. Wang et al. introduced an AutoML architecture for a blockchain-focused approach [810]. It comprises of six strands: (i) Association 
www.rspsciencehub.com

layer (ii) Clients layer, (iii) Implementation layer (iv) AutoML layer (vi) Blockchain layer (v) Data transmission layer. This system allows entities to maintain their information confidentially, simplify their operations and exchange information in a jointly advantageous and secure manner with certain entities. An information supplier, an information client, and a business analyst in the system. Various mechanisms enable interactions among the supplier, the user and the administrator like configuration, log, transaction and acknowledgment guidelines and query procedure.

\subsection{System Customization}

System customization is an aspect that utilizes forecasts through smart machines to enhance product performance, such as user acts, smart data collection, and message pad composition. The log information attempts to transform the machine's service.

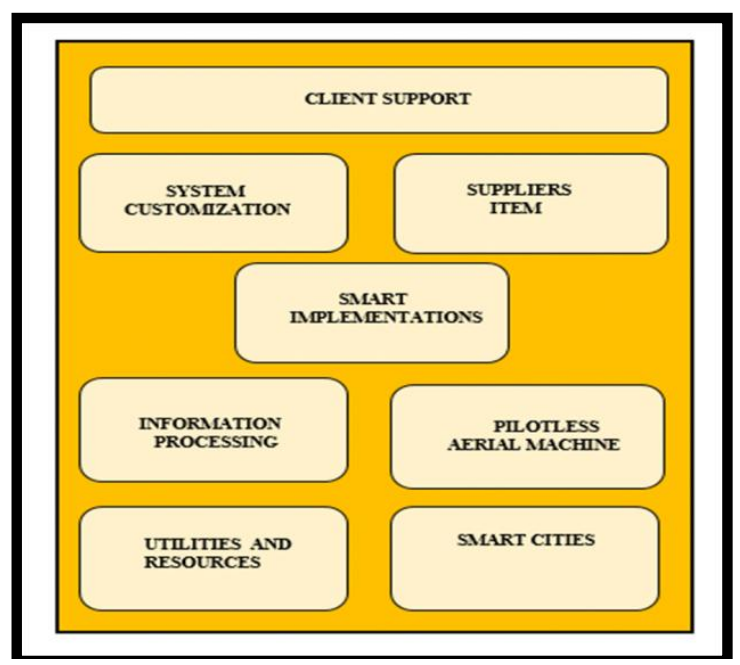

Fig 4. Smart Implementation

\subsection{Suppliers Item}

Entities have begun to believe blockchaincentered practices to encourage manufacturing, safety, accountability and regulatory inspections as a function of the production cycle. To move information quite efficiently and easily, the firm uses blockchain software giving its consumer's sense of security, independence of loading, paying and connection to the car from third parties.

\subsection{Information Processing}

Information Processing is a revolutionary forum
Volume 02 Issue 09 September 2020

for retail investors from all across the globe that allows sophisticated investing feasible. This platform centered on Ethereum has been introduced that has developed at a similar moment in eliminating fixed-point loss and safeguarding data. There are three bodies, an information supplier, an information client, and a business analyst in the system. Various mechanisms enable interactions among the supplier, the user and the administrator like configuration, log, transaction and acknowledgment guidelines and query procedure.

\subsection{Pilotless Aerial Machine}

An airplane operating without an operator (human) on-site is a UAV or drone. Kuzmin et al. suggested a blockchain-centered UAV Net system that involves multiple types of equipment such as a satellite network, mobile base centers and ground-based facilities [2]. This approach utilized consent evidence of graph method with a generalized restricted memory protocol of any current quickest route to validate a current payment.

\subsection{Smart Cities}

The everyday environments of people are changed by smart cities. ML and blockchain disruptive developments serve a key part in smart city development to provide essential amenities and network elements such as medical care, city management, smart buildings, education, transport, property development and necessities. (i) Smart Homes: It is possible to track smart homes and system personalization may enhance the standard of living. (ii) Smart Parking Method: Here, the entrance and exit of automobiles can be monitored in a supervised way inside the smart city for the various park areas feasible [9].

(iii) Smart Vehicle Traffic: Vehicle traffic information with an effective review would be of considerable importance to the community and customers [10]. By employing this information, everybody can determine the period of entry to a location. (iv) Monitoring devices: physical protection is of paramount importance to people wherever they reside. Smart equipment such as ML and BT can be designed to tackle this 
www.rspsciencehub.com

problem. Subsequently, one of the complicated activities is to analyze and gather information and identify incidents. (v) Smart Management and Smart Education: A city can be skillfully maintained through intelligent management. It took forecasts and analysis to maintain the material up to the level.

\subsection{Utilities and Resources}

In the energy sector, BT helps optimize the sharing of resources. For instance, by incorporating BT, IOT allows for smart transition through the whole power sector. It utilizes the idea of the creation and usage of peer-to-peer resources. Through enabling the peer-to-peer (P2P) platform for consumers, BT has indeed disrupted the platform. The monetary transition utilizing the globe of digital currency is observed; additionally, because of the merging ML and BT many technology transformations will be experienced.

\section{Anticipated Investigation Problems and Concerns}

Investigators are moving ahead to such techniques internationally, but the convergence of BT and ML often avoids several barriers. Their convergence is indeed in its development. There are several unanswered concerns and problems to be resolved. Here, as illustrated in diagram 5, we address the revolutionary accessible problems and concerns of ML implementation in BT for safe interaction. We have outlined the difficulties such as feasibility, structure, safety, storage, deployment, safeguarding and responsiveness to quantity.

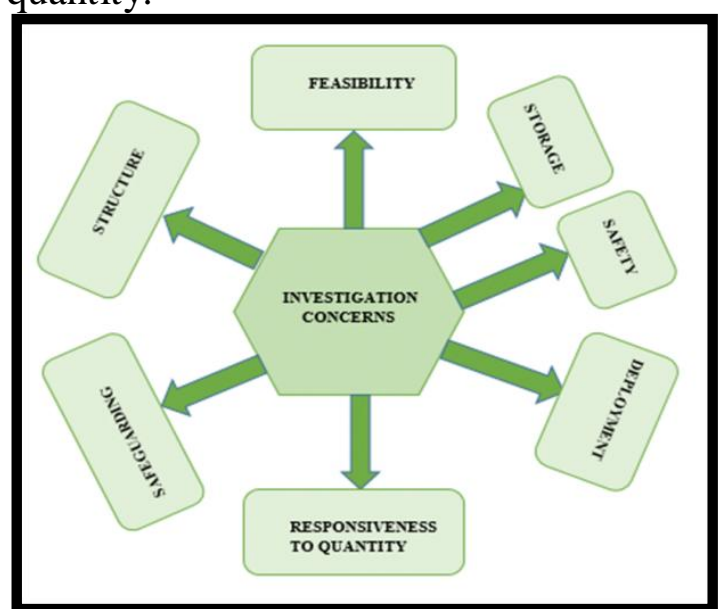

Fig 5. Anticipated Investigation Problems and Concerns.

10.1 Feasibility
Volume 02 Issue 09 September 2020

If the source of data may not be assured and many organizations are prominent in the

decentralized framework, the blockchain is a feasible option. If the output is needed then the best choice is a basic repository. Its design can be considered in every framework before its usage.

\subsection{Structure}

This may entail management of the system, processing hardware, distributed space and standards for interaction. Items designed for usage in the blockchain are also under examination, though.

\subsection{Safety}

All blockchain entities have access to information created by machines to be processed on it. This contributes to a possible issue for information protection that must be held either secret or confidentiality.

\subsection{Storage}

The Blockchain scale is continuing to expand as additional nodes are introduced to it. A rise in the chain size also affects efficiency.

\subsection{Deployment}

An equally massive amount of payments would involve a broad-scale blockchain infrastructure. Thus, to satisfy the imminent request, the inclusion of blocks and exchanges requires to be reduced.

\subsection{Safeguarding}

Blockchain are distributed and open to security threats. The main significant issue is that the consent mechanism may be breached due to threats, so the processing capacity of several farms determines that frames are connected to the system.

\subsection{Responsiveness to Quantity}

Quantum machines will soon break down the hashing methods employed by blockchain. This is primarily at stake, as it requires one-way encryption methods. An analysis is undergoing to build a particle-computing blockchain.

\section{Conclusion}

Current blockchain and ML innovations have 
www.rspsciencehub.com

rendered them transformative developments. Different smart technologies such as smart cities, $\mathrm{UAV}, \mathrm{SG}$, information mining will work as an integral part of the decentralized network. ML-BT approach, preventive measures and measurements of the smart implementation are introduced. A comprehensive review of methods and techniques accessible for each category is provided. Then throughout ML acceptance in BT-focused machines, the numerous analysis challenges facing the entail remedies are reported. A range of research opportunities like the accessibility of connectivity, fundamental efficiency, and privacy concerns that can function as potential paths for analysis in this area is highlighted and concluded in the report.

\section{Acknowledgement}

Every achievement needs several individuals' dedication so this task is not exceptional. Besides, it is indeed my primary responsibility to honor the individuals that guided and supported me to conduct this research report. Therefore, I seize the privilege to sincerely appreciate my grandma Shardaba during the task for her outstanding support and inspiration. I would like to express my sincere thanks to my parents: Dansinh and Asha Solanki and all my family members, without their encouragement my research might not have become what it is.

\section{Reference}

[1].U. Bodkhe, P. Bhattacharya, S. Tanwar, S. Tyagi, N. Kumar and M. S. Obaidat, "Blohost: Blockchain-enabled smart tourism and hospitality management," in Proc. International Conference Computation, Information Telecommunication System (CITS), Aug. 2019, pp. 1-5.

[2].A. Kuzmin and E. Znak, "Blockchainbased structures for a secure and operate a network of semi-autonomous unmanned aerial vehicles," in Proc. IEEE International Conference Service Operation Logistics, Information (SOLI), July 2018, pp. 32-37.

[3].D. Ucci, L. Aniello, and R. Baldoni,
Volume 02 Issue 09 September 2020

"Survey of machine learning techniques for malware analysis,' Computer Security, vol. 81, pp. 123-147, Mar. 2019.

[4]. K. Salah, M. Rehman, N. Nizamuddin, and A. Al-Fuqaha, "Blockchain for AI: Review and open research challenges,' IEEE Access, vol.7, pp. 10127-10149, 2019.

[5].P. Louridas and C. Ebert, "Machine Learning," IEEE Software, vol. 33, no. 5, pp. 110-115, May 2016.

[6].R. Saravanan and P. Sujatha, "A state of art techniques on machine learning algorithms: A perspective of supervised learning approaches in data classification," in Proc. 2nd International Conference Intelligence Computation Control System (ICICCS), Jun. 2018, pp. 945-949.

[7].R.-Y. Chen, “A traceability chain algorithm for artificial neural networks using $\mathrm{T}-\mathrm{S}$ fuzzy cognitive maps in blockchain,' Future Generation Computer System, vol. 80, pp. 198-210, Mar. 2018.

[8].W. M. Wang, H. Guo, Z. Li, Y. Shen, and A. V. Barenji, "towards open and automated customer service: A blockchain-based automl framework," in Proc. 2nd International Conference on Computer Science Application Engineering (CSAE), New York, NY, USA, 2018, pp. 27:1-27:6.

[9].A. Kumari, S. Tanwar, S. Tyagi, N. Kumar, M. Maasberg and K.-K. R. Choo, "Multimedia big data computing and Internet of Things applications: A taxonomy and process model,' J. Network Computer Application, vol. 124, pp. 169195, 2018.

[10].A. Mewada, S. Tanwar, and Z. Narmawala, "Comparison and evaluation of real time reservation technologies in the intelligent public transport system," in Proc. 5th Int. Conf. Parallel, Distributed Grid Computing (PDGC), Dec. 2018, pp. 800-805. 